$\mathrm{ETH} / \mathrm{TH} / 95-28$

23 October 1995

\title{
The Scalar Potential in Noncommutative Geometry
}

\author{
A. H. Chamseddine \\ Theoretische Physik \\ ETH-Hönggerberg \\ CH-8093, Zürich \\ Switzerland
}

\begin{abstract}
We present a derivation of the general form of the scalar potential in Yang-Mills theory of a non-commutative space which is a product of a four-dimensional manifold times a discrete set of points. We show that a non-trivial potential without flat directions is obtained after eliminating the auxiliary fields only if constraints are imposed on the mass matrices utilised in the Dirac operator. The constraints and potential are related to a prepotential function.
\end{abstract}


One of the most attractive aspects of noncommutative geometry [1-2] is that it deals with spaces which could not be handled otherwise. It also offers tools which could be used in probing the hidden structure of space-time [3]. An encouraging indication of the relevance of this new geometry to particle physics is the fact that the simplest noncommutative space one considers, where the algebra is $C^{\infty}(M) \otimes\left(M_{1}(C) \oplus M_{2}(C) \oplus\right.$ $M_{3}(C)$ ) reproduces, classically, the standard model with all of its details [4-5]. It also provides an appealing geometrical picture for the Higgs field. These ideas were then applied to grand unified theories [6] and it was found that the main advantage of this scheme is that the composition of the Higgs sector is almost uniquely fixed. This is in complete contrast to the usual treatment of grand unified theories where the advantages gained in reduction of the fermionic representations and the unification of the coupling constants are wiped out by the arbitrariness in the Higgs fields representations. What is still missing in noncommutative geometry is the identification of the hidden new symmetries present as this is needed to protect, at the quantum level, any classical predictions [7]. A step in this direction has recently been made where it was conjectured that the hidden symmetry of the noncommutative standard model is the quantum group $S U(2)_{q}$ at the cubic root of unity [8].

It is therefore important to simplify the calculations involved in deriving a general formula for the Yang-Mills noncommutative action associated with a product space of a continuous manifold times a discrete set of points, with an appropriate fermionic representation. This program was started in [6] but the result involved auxiliary fields which have to be eliminated. This last step was only carried out for some specific models, and it was found that the form of the potential depends on the mass matrices used in the Dirac operator. These mass matrices also turn out to be the vevs (vacum expectation values ) of the Higgs fields and must satisfy certain conditions. The purpose of this letter is to derive a general form of the potential, after elimination of the auxiliary fields, and to determine the constraints that must be imposed on the mass matrices in the Dirac operator to obtain a physically acceptable potential.

We start with the spectral triple $(\mathcal{A}, h, D)$, where $h$ is a Hilbert space, $\mathcal{A}$ is an involutive algebra of operators on $h$, and $D$ is an unbounded self-adjoint operator on $h$ [1]. Let $M$ be a compact Riemannian spin-manifold, $\mathcal{A}_{1}$ the algebra of functions on $M$, and $\left(h_{1}, D_{1}, \Gamma_{1}\right)$ the Dirac- $K$ cycle with $h_{1} \equiv L^{2}\left(M, \sqrt{g} d^{d} x\right)$ on $\mathcal{A}_{1}$. Let $\left(\mathcal{A}_{2}, h_{2}, D_{2}\right)$ be given by $\mathcal{A}_{2}=\oplus_{p=n_{1}}^{n_{N}} M_{p}(C)$, where $M_{p}(C)$ is the set of all $p \times p$ matrices and $h_{2}=\oplus_{p=n_{1}}^{n_{N}} h_{2, p}$ where $h_{2, p}$ is the Hilbert space $C^{p}$. We take $\mathcal{A}$ and $D$ to be

$$
\begin{aligned}
& \mathcal{A}=\mathcal{A}_{1} \otimes \mathcal{A}_{2} \\
& D=D_{1} \otimes 1+\Gamma_{1} \otimes D_{2} .
\end{aligned}
$$

To every $a \in \mathcal{A}$ we associate a N-plet $\left(a_{n_{1}} \cdots a_{n_{N}}\right)$ of matrix-valued functions on $M$, where $a_{p}$ are $p \times p$ matrix valued functions. In this decomposition, the operator $D$ 
becomes

$$
D=\left(\begin{array}{cccc}
\not \partial \otimes 1_{n_{1}} \otimes 1_{3} & \gamma_{5} \otimes M_{12} \otimes K_{12} & \ldots & \gamma_{5} \otimes M_{1 N} \otimes K_{1 N} \\
\gamma_{5} \otimes M_{21} \otimes K_{21} & \not \partial \otimes 1_{n_{2}} \otimes 1_{3} & \ldots & \gamma_{5} \otimes M_{2 N} \otimes K_{2 N} \\
\vdots & \vdots & \ddots & \vdots \\
\gamma_{5} \otimes M_{N 1} \otimes K_{N 1} & \gamma_{5} \otimes M_{N 2} \otimes K_{N 2} & \ldots & \not \partial \otimes 1_{n_{N}} \otimes 1_{3}
\end{array}\right)
$$

where $M_{m n}^{*}=M_{n m}$ and $m, n=1, \cdots, N, m \neq n$ and the $K_{m n}$ are generation mixing $3 \times 3$ matrices.

Let $E$ be a vector bundle characterized by the vector space $\mathcal{E}$ of its sections. We shall consider the example where $\mathcal{E}=\mathcal{A}$. Let $\rho$ be a self-adjoint element in the space, $\Omega^{1}(\mathcal{A})$, of one forms

$$
\rho=\sum_{i} a^{i} d b^{i}
$$

where $\Omega \cdot(\mathcal{A})=\oplus_{n=0}^{\infty} \Omega^{n}(\mathcal{A})$ is the universal differential algebra, with $\Omega^{0}(\mathcal{A})=\mathcal{A}$ [1]. An involutive representation of $\Omega \cdot(\mathcal{A})$ is provided by the map $\pi: \Omega \cdot(\mathcal{A}) \rightarrow B(h)$ defined by

$$
\pi\left(a_{0} d a_{1} \ldots d a_{n}\right)=a_{0}\left[D, a_{1}\right]\left[D, a_{2}\right] \ldots\left[D, a_{n}\right],
$$

where $B(h)$ is the algebra of bounded operators on $h$. The image of the one-form $\rho$ is

$$
\pi(\rho)=\sum_{i} a^{i}\left[D, b^{i}\right]
$$

and this takes the matrix form:

$$
\pi(\rho)=\left(\begin{array}{cccc}
\gamma^{\mu} \otimes A_{\mu 1} \otimes 1_{3} & \gamma_{5} \otimes \phi_{12} \otimes K_{12} & \ldots & \gamma_{5} \otimes \phi_{1 N} \otimes K_{1 N} \\
\gamma_{5} \otimes \phi_{21} \otimes K_{21} & \gamma^{\mu} \otimes A_{\mu 2} \otimes 1_{3} & \ldots & \gamma_{5} \otimes \phi_{2 N} \otimes K_{2 N} \\
\vdots & \vdots & \ddots & \vdots \\
\gamma_{5} \otimes \phi_{N 1} \otimes K_{N 1} & \gamma_{5} \otimes \phi_{N 2} \otimes K_{N 2} & \ldots & \gamma^{\mu} \otimes A_{\mu N} \otimes 1_{3}
\end{array}\right)
$$

where the new variables $A_{\mu m}$ and $\phi_{m n}$ are functions of the $a^{i}$ and $b^{i}$ given by

$$
\begin{aligned}
& A_{\mu m}=\sum_{i} a_{m}^{i} \partial_{\mu} b_{m}^{i}, \quad m=1,2, \ldots, N, \\
& \phi_{m n}=\sum_{i} a_{m}^{i}\left(M_{m n} b_{n}^{i}-b_{m}^{i} M_{m n}\right), \quad m \neq n,
\end{aligned}
$$

and satisfy $A_{\mu m}^{*}=-A_{\mu m}$ and $\phi_{m n}^{*}=\phi_{n m}$. The two-form $d \rho$ is:

$$
d \rho=\sum_{i} d a^{i} d b^{i}
$$

and its image under the involutive representation $\pi$ is given by

$$
\pi(d \rho)=\sum_{i}\left[D, a^{i}\right]\left[D, b^{i}\right]
$$


The curvature $\theta$ is defined by

$$
\theta=d \rho+\rho^{2} .
$$

It must be noted that the representation $\pi$ is ambiguous [4], a fact that will explain the appearence of auxiliary fields. This can be seen from the fact that if $\pi(\rho)$ is set to zero, $\pi(d \rho)$ is not necessarily zero, and the correct space of forms to work on is $\Omega_{D}(\mathcal{A})=\oplus \Omega_{D}^{n}(\mathcal{A})$, where $\Omega_{D}^{n}(\mathcal{A})=\frac{\Omega^{n}(\mathcal{A})}{(\operatorname{Ker} \pi+d \operatorname{Ker} \pi)_{n}}$, where $\operatorname{Ker} \pi$ is the kernel of the map $\pi$.

The representation of the curvature $\pi(\theta)$ can be written in terms of components. First, the diagonal elements are given

$$
\pi(\theta)_{m m}=\frac{1}{2} \gamma^{\mu \nu} F_{\mu \nu}^{m}+\left(\sum_{p \neq m}\left(\left|K_{m p}\right|^{2}\left(\left|H_{m p}\right|^{2}-\left|M_{m p}\right|^{2}\right)\right)-X_{m m}^{\prime} \quad m=1,2 \ldots, N,\right.
$$

where we have defined

$$
\begin{aligned}
X_{m m}^{\prime} & \left.=\sum_{i} a_{m}^{i} \not^{2} b_{m}^{i}+\left(\partial^{\mu} A_{\mu}^{m}+A^{\mu m} A_{\mu}^{m}\right)+\left[\sum_{n \neq m} a_{m}^{i}\left|K_{m n}\right|^{2} M_{m n} M_{n m}, b_{m}^{i}\right]\right) \\
F_{\mu \nu} & =\partial_{\mu} A_{\nu}^{m}-\partial_{\nu} A_{\mu}^{m}+\left[A_{\mu}^{m}, A_{\nu}^{m}\right] \\
H_{m p} & =\phi_{m p}+M_{m p} .
\end{aligned}
$$

The non-diagonal elements of $\pi(\theta)$ are given by $(m \neq n)$ :

$$
\begin{aligned}
\pi(\theta)_{m n}=- & \gamma_{5} K_{m n}\left(\not \partial H_{m n}+A_{m} H_{m n}-H_{m n} A_{n}\right) \\
& +\sum_{p \neq m, n} K_{m p} K_{p n}\left(H_{m p} H_{p n}-M_{m p} M_{p n}\right)-X_{m n} .
\end{aligned}
$$

The curvature $\theta$ is self-adjoint: $\pi(\theta)_{m n}^{*}=\pi(\theta)_{n m}$.

The full noncommutative action is given by

$$
\begin{aligned}
I= & \int d^{4} x\left(-\frac{1}{4} \sum_{m} F_{\mu \nu}^{m} F^{\mu \nu m}\right. \\
& \left.+\sum_{p \neq m}\left|\partial_{\mu} H_{m p}+A_{\mu m} H_{m p}-H_{m p} A_{\mu p}\right|^{2}-V\right) \\
& +(\Psi,(D+\pi(\rho)) \Psi),
\end{aligned}
$$

where the fermions are collectively denoted by $\Psi$, and the scalar potential by $V$. The fields $X_{m n}^{\prime}$ and $X_{m n}$ are not all independent, and the relations among them depend on the structure of the mass matrices $M_{m n}$. We would like to find out what sort of constraints must be imposed on the mass matrices in order to get a non-trivial scalar potential. In the generic case where these matrices are arbitrary, and the dimensions of all the matrices are different than one, every term contributing to the potential in 
the curvature will be moded out. To see this explictely, we note that the potential is given by

$$
\begin{aligned}
V & \left.=\left.\left|\sum_{p \neq m}\right| K_{m p}\right|^{2}\left(\left|H_{m p}\right|^{2}-\left|M_{m p}\right|^{2}\right)-X_{m m}^{\prime}\right)\left.\right|^{2} \\
& \left.+\sum_{n \neq m} \sum_{p \neq m, n}\left|K_{m p} K_{p n}\left(H_{m p} H_{p n}-M_{m p} M_{p n}\right)-X_{m n}\right|^{2}\right),
\end{aligned}
$$

which is a sum of squares. Each of these terms contain an auxiliary field, and when all are eliminated the potential vanishes. Therefore, for the matrices $M_{m n}$ to correspond to minima of the potential they must sastisfy certain conditions. We shall now determine the necessary constraints.

The space of auxiliary fields is found by calculating the kernel of the operator $\left.\pi(d \rho)\right|_{\pi(\rho)=0}$. A simple calculation gives

$$
\begin{aligned}
(\text { Aux })_{m m} & =\sum_{i} a_{m}^{i} \partial^{2} b_{m}^{i}+\left[\sum_{n \neq m} a_{m}^{i}\left|K_{m n}\right|^{2} M_{m n} M_{n m}, b_{m}^{i}\right] \\
(\mathrm{Aux})_{m n} & =\sum_{i} a_{m}^{i} \sum_{p \neq m, n} K_{m p} K_{p n}\left(M_{m p} M_{p n} b_{n}^{i}-b_{m}^{i} M_{m p} M_{p n}\right), \quad m \neq n,
\end{aligned}
$$

Equation (16) is a function of the square of the matrices $M_{m n}$ and as these in general are not equal to a linear combination of themselves, the auxiliary fields produced will be nonconstrained scalar functions. When the curvature two-form is moded out by the auxiliary fields along diagonal and non-diagonal terms, the scalar parts of the curvature drop out. Therefore there is a need to carefully chose the matrices $M_{m n}$ in order to get an acceptable scalar potential.

The simplest possibility corresponds to the case where the dimension of one of the matrices, e.g. $n_{1}$ is equal to one. Then the commutator in (Aux) ${ }_{11}$ drops out, and it reduces to $(\mathrm{Aux})_{11}=\sum_{i} a_{1}^{i} \not^{2} b_{1}^{i}$. After elimination of this field, the contribution of the first diagonal part of the curvature to the potential reduces to the form:

$$
v_{11}=\sum_{p \neq 1}\left(\left|K_{1 p}\right|^{2}\right)^{\perp}\left(\left|H_{1 p}\right|^{2}-\left|M_{1 p}\right|^{2}\right),
$$

where

$$
\left(\left|K_{m p}\right|^{2}\right)^{\perp}=\left|K_{m p}\right|^{2}-\operatorname{Tr}\left(\left|K_{m p}\right|^{2}\right) 1_{3},
$$

and where we have normalised the trace so that $\operatorname{Tr}(1)=1$. Therefore, whenever the dimension of one of the matrix algebras is one, there is no need to impose any conditions on the mass matrices $M_{1 p}$ for the potential to survive. This is the situation encountered in the standard model where the dimension of one of the matrix algebras is one.

In all other cases we have to restrict the choice of the mass matrices so that some of the auxiliary fields could be expressed in terms of the Higgs fields, and this would 
make them linearly dependent. As the potential is the sum of squares, its minimum occurs when each term vanishes. Each of these terms is quadratic in the fields, so the minimum values of the Higgs fields $H_{m p}$ must satisfy quadratic equations.

First, we have to assume that the generation mixing matrices are related. The simplest possibility is

$$
\left|K_{m n}\right|^{2}=|K|^{2} \quad \forall m, n,
$$

This condition could be slightly relaxed, but the analysis will become less transparent. The most general constraint on the matrices $M_{m n}$ is then:

$$
\sum_{p \neq m \neq n} M_{m p} M_{p n}=\alpha_{m n} M_{m n}
$$

Using equations (18) and (19) it is easy to show that the fields $X_{m n}$ reduce to

$$
X_{m n}=-|K|^{2} \alpha_{m n} \phi_{m n},
$$

and the terms $v_{m n}$ in the potential (the potential is a function of the square of $v_{m n}$ ) simplify to the nice form

$$
v_{m n}=|K|^{2}\left(\sum_{p \neq m \neq n} H_{m p} H_{p n}-\alpha_{m n} H_{m n}\right) .
$$

It is clear that the equations of motion satisfied by $H_{m n}$ are the same as that satisfied by the mass matrices $M_{m n}$. In other words the fields $\phi_{m n}$ have zero vevs. The other contributions to the potential from $v_{m m}$ do not survive as there is no possible simplification for $X_{m m}^{\prime}$. When $X_{m m}^{\prime}$ is eliminated the parts coming from the diagonal components of the curvature disappear. Therefore, except when $\operatorname{dim}\left[n_{m}\right]=1$, the terms in the potential dependent on $v_{m m}$ vanish. We can understand this result by studying the gauge transformations of the Higgs fields. From the gauge transformations of the curvature

$$
\theta \rightarrow{ }^{g} \theta=g \theta g^{*}
$$

where $g$ is an element of $\mathcal{A}$ with the representation

$$
g \rightarrow \operatorname{diag}\left(g_{1}, \ldots, g_{N}\right)
$$

we can show that

$$
{ }^{g} H_{m n}=g_{m} H_{m n} g_{n}^{*} \quad m \neq n,
$$

Equation (24) then implies that

$$
\sum_{p} H_{m p} H_{p n} \rightarrow g_{m}\left(\sum_{p} H_{m p} H_{p n}\right) g_{n}^{*}
$$

and transforms in the same way as $H_{m n}$ allowing for constraints to be imposed relating $\sum_{p} H_{m p} H_{p n}$ to $H_{m n}$. On the other hand no such relation is possible for $\sum_{p} H_{m p} H_{p m}$ as this combination transforms as

$$
g_{m}\left(\sum H_{m p} H_{p m}\right) g_{m}^{*}
$$


and there is no Higgs field with such a transformation. We already noted that the potential is a sum of squares, a feature which is also present in globaly supersymmetric theories. There, one can define a gauge invariant superpotential $g\left(z^{i}\right)$, holomorphic in the complex scalar fields $z^{i}$, and the potential takes the very elegant form

$$
V=\left|\frac{\partial g(z)}{\partial z^{i}}\right|^{2}
$$

By analogy, we can define here a prepotential gauge invariant function

$$
\left.g(H)=\operatorname{tr}\left(\frac{1}{3} \sum_{p \neq m \neq n} H_{m p} H_{p n} H_{n m}-\frac{1}{2} \sum_{m, n} \alpha_{m n} H_{m n} H_{n m}\right)\right) .
$$

The total potential in this case can be written in the simple form:

$$
V=\left(\operatorname{Tr}|K|^{4}\right)^{\perp} \sum_{m, n}\left|\frac{\partial g}{\partial H_{m n}}\right|^{2}
$$

provided that non of the matrix algebras have dimension one. In equation (29) we have denoted

$$
\left(\operatorname{Tr}|K|^{4}\right)^{\perp}=\operatorname{Tr}\left(|K|^{4}\right)-\left(\operatorname{Tr}|K|^{2}\right)^{2} .
$$

The fact that there are no contributions to the scalar potential from the diagonal parts of the curvature is not very desirable, as there will be many Higgs fields whose vevs are left undetermined. To avoid this and to make sure that the potential has no flat directions we consider the following situation. By imposing a permutation symmetry on two points (e.g. $1 \leftrightarrow 2$ ), we can have Higgs fields belonging to the adjoint representation of the gauge group. This makes it possible to impose more constraints on the mass matrices. This case is very important for model building as adjoint representations are important for symmetry breaking at high energies. From the permutation symmetry we deduce that $M_{12}=M_{21}$, and $a_{1}=a_{2}$. This in turn implies that $A_{\mu 1}=A_{\mu 2}$ and $H_{12}=H_{21}=\Sigma$. It follows that

$$
H_{12} H_{21} \rightarrow g_{1} H_{12} H_{21} g_{1}^{*}
$$

where we have used $g_{1}=g_{2}$. With this it is now possible to impose conditions of the form

$$
\sum_{p \neq m} M_{1 p} M_{p 1}=\alpha_{1} M_{12}+\beta_{1} 1_{n_{1}}
$$

Such conditions could be generalised whenever two points $m$ and $n$ have a permutation symmetry. In this case we write

$$
\sum_{p \neq m} M_{m p} M_{p m}=\alpha_{m} M_{m n}+\beta_{m} 1_{n_{m}}
$$

Using equations (18) and (31) in (12) it is easily verified that $X_{m m}^{\prime}$ simplifies to

$$
\begin{gathered}
X_{m m}^{\prime}+\sum_{p \neq m}\left|M_{m p}\right|^{2}=\sum_{i} a_{m}^{i} \not b_{m}^{i}+\left(\partial^{\mu} A_{\mu m}+A^{\mu m} A_{\mu m}\right) \\
+\alpha_{m} \Sigma_{m}+\alpha_{m} \Sigma_{m}+\beta_{m} 1_{n_{m}}
\end{gathered}
$$


where we have denoted $H_{m m}$ by $\Sigma_{m}$. This implies that the diagonal components of $v_{m m}$, after projecting the kernel of the Dirac operator, reduce to

$$
v_{m m}=\left(|K|^{2}\right)^{\perp}\left(\sum_{p \neq m}\left|H_{m p}\right|^{2}-\alpha_{m} \Sigma_{m}-\beta_{m} 1_{n_{m}}\right) .
$$

The contributions of the diagonal parts of the curvature to the potential will be the square of such terms. The presence of the new terms in the potential is a reflection of the fact that new terms to the prepotential could be added. The most general gauge invariant prepotential in this case is given by

$$
\begin{aligned}
g & =\operatorname{tr}\left(\frac{1}{3}\left(\sum_{m} \Sigma_{m}^{3}+\sum_{p \neq m, n} H_{m p} H_{p n} H_{n m}\right)\right. \\
& \left.-\frac{1}{2} \sum_{m}\left(\alpha_{m} \Sigma_{m}^{2}+\sum_{p \neq n} \alpha_{m p} H_{m p} H_{p m}\right)-\beta_{m} \Sigma_{m}\right) .
\end{aligned}
$$

We are now in a position to write down the most general physically acceptable potential that results from the Yang-Mills noncommutative action. Assuming we have s of the $n_{m} \times n_{m}$ matrices to be of dimension one, and $\mathrm{r}$ couple of points have permutation symmetry, the scalar potential will be given by

$$
\begin{aligned}
V & =\operatorname{Tr}\left(|K|^{4}\right)^{\perp}\left(\sum_{s}\left(\sum_{p}\left(\left|H_{s p}\right|^{2}-\left|M_{s p}\right|^{2}\right)\right)^{2}\right. \\
& \left.+\sum_{r}\left(\left|\Sigma_{r}\right|^{2}-\alpha_{r} \Sigma_{r}-\beta_{r} \Sigma_{r}+\sum_{p \neq s}\left|H_{r p}\right|^{2}\right)^{2}\right) \\
& +\operatorname{Tr}|K|^{4} \sum_{m, n \neq r} \sum_{p \neq m, n}\left|H_{m p} H_{p n}-\alpha_{m n} H_{m n}\right|^{2} .
\end{aligned}
$$

Naturally this formula could be reexpressed in terms of the gauge invariant prepotential $g$ :

$$
V=\operatorname{Tr}\left(|K|^{4}\right)^{\perp} \sum_{r}\left|\frac{\partial g}{\partial \Sigma_{r}}\right|^{2}+\operatorname{Tr}|K|^{4} \sum_{p \neq r}\left|\frac{\partial g}{\partial H_{m p}}\right|^{2},
$$

The fact that the potential is a sum of squares and is derivable from a prepotential makes it as near to supersymmetric theories as possible without requiring supersymmetric partners. However, they are not in general supersymmetric, except in certain cases related to $N=2$ and $N=4$ supersymmetry [9]. This, however, could be a signal of the presence of new kind of symmetries, such as quantum symmetries [8].

We conclude by noting that this derivation takes the pain of obtaining the general form of the lagrangian for a unified theory based on noncommutative geometry. Once the spectral triple and the symmetries of the matrix algebras are specified, the potential could immediately be written. This will make constructing new realistic models based on noncommutative geometry easier to achieve. It is quite important to derive the new models using the anticipated quantum symmetries, in such a way that any predictions made could be protected at the quantum level by these symmetries. 


\section{Acknowledgments}

I would like to thank Alain Connes, Jüerg Fröhlich, Olivier Grandjean, Daniel Kastler and Daniel Testard for stimulating discussions. I would also like to thank the Erwin Schrödinger International Institute for Mathematical Physics, Vienna, for hospitality where this work was done.

\section{References}

[1] A. Connes, Publ. Math. IHES 62 (1983) 44;

in the interface of mathematics and particle physics, Clarendon press, Oxford 1990, Eds D. Quillen, G. Segal and S. Tsou;

Noncommutative Geometry, Academic Press, N.Y. (1994).

[2] M. Dubois-Violette, C.R. Acad. Sci. Paris, 307 (1988) 403;

M. Dubois-Violette, R. Kerner and J. Madore, J. Math. Phys. 31 (1990) 316; Class. Quant. Grav. 6 (1989) 1709.

[3] A. H. Chamseddine and J. Fröhlich in Yang-Festschrift editors C. S. Liu and S.-T. Yau.

[4] A. Connes and J. Lott,Nucl.Phys.B Proc.Supp. 18B (1990) 29, North-Holland, Amsterdam;

in Proceedings of the 1991 Summer Cargèse Conference edited by J. Fröhlich et al (Plenum, New York, 1992).

[5] D. Kastler, Rev. Math. Phys. 5 (1993) 477;

D. Kastler and T. Schücker, Theor. Math. Phys. 92 (1992) 522;

B. Iochum, D. Kastler and T. Schücker, Marseille preprint, CPT-95/P.33197, and hep-th/9507150.

[6] A. H. Chamseddine, G. Felder and J. Fröhlich, Phys. Lett. B296 (1992) 301; Nucl.Phys. B395 (1993), 672;

A. H. Chamseddine and J. Fröhlich, Phys. Rev. D50 (1994) 2893.

[7] E. Alvarez, J. M. Garcia-Bondia, and C. P. Martin, Phys. Lett. B306 (1993) 55.

[8] A. Connes, Plenary Talk Presented at the Trest Meeting on Noncommutative Geometry May 1995.

[9] A. H. Chamseddine Phys. Lett. B332 (1994) 349. 